\title{
Application of Schema Theory in Painting Modeling
}

\author{
Bao Liu \\ Animation Teaching and Research Office \\ Art and Design Department \\ College of Hu manities \\ Yantai Nanshan University \\ Yantai, China 265713
}

\begin{abstract}
This paper starts with the abnormal phenomena in the current situation of domestic painting education, through analysis, it points out that the underying cause beneath these phenomena is the lack of schema in the author's mind. It then further expounds the raising background of schema as well as characteristics and relevance of schematism, indicating the important role of schema theory in the development of contemporary painting education.
\end{abstract}

Keywords—schema; epistemology; art education

\section{INTRODUCTION}

When it comes to painting, people generally admire and commend the maestros or good painters, besides, they will subconsciously form such an impression: "This person is truly talented"; That is a common reaction from a layman's point of view on painting by painters, actually one will be considered as being talented whichever vocation he is engaged in as long as he is outstanding and perfect. For such "art innatism", most people are taking a tacit attitude, which naturally wrapping a mysterious veil of talent on artists. This phenomenon has its historical origins, the view of innatism in literary literature theory has caused philosophers' attention, a lot of discussions have been carried out in this proposition.

\section{VICIOUS CYCLE IN ART EDUCAT ION}

Throughout our generation and the contemporary art education, most of the art education workers will tell their students earnestly their small problems in painting a shape during instruction; the author belongs to the generation after 80 s, hence I clearly remember I myself being told the problems by my teacher. This is certainly not negating the teachers and their tuition, but that was how teachers instructed students, more sadly, most art education workers after 80 s are imitating their teachers and teaching their own students in this way. Through reflection on the four generations of teachers' teachers, one's own teachers, one's own and one's own students, shouldn't one ask whether such education the working method is scientific and reasonable? Doesn't anyone feel that such education the working method merely teaches students superficial knowledge? However, there is existence of such education and teaching method in many specialties of fine arts in domestic universities. The author also found in actual teaching activities that, regardless how hard the teacher teaches and student learns, students still feel hard to improve animation modeling in a short time, because the specialty of animation has too many images need to be known, with a very broad covering range, hence the teaching effect is not very good, especially the modeling of cartoon image design, the above teaching methods require our further reflection.

\section{APPLICATION OF SCHEMA BY CHINESE LANDSCAPE PAINTERS OR FLOWER AND BIRD PAINTERS}

Chinese landscape painters or flower and bird painters are often able to quickly paint mass beautiful rivers and mountains of a country, birds and insect on a fair and clear Chinese art paper without going outdoors or reference materials, this is not a "talent", rather this phenomenon has underlying causes behind. As Gombrich said: "Chinese artists are still the "creators" of mountains, trees or flowers nowadays, and they can imagine because they know the secret about their existence, but what they do is to record and evoke a mental state, and this state of mind is deeply rooted in Chinese ideas about the nature of the universe." [1]

Do Chinese artists know the "secret" about their existence? What inspiration and enlightenment will this secret have on our art education, learning of cartoon image design, modeling of specialty of animation or teaching of commercial art education, they all need our further study. The author summarizes the "secret" as "schema", which can explain the "painting innatism" and "modern art education malpractice" problems in F.C.Ayer's doctoral thes is on painting psychology referenced by Gombrich. "A well-trained painter will learn plenty of schema, according to these schema he could quickly draw the schema of an animal, a flower or a house on paper. This can be used to reproduce the supporting points of his me mory image, and then he gradually rectify the schema, until meeting what he wants to express. Many good painters who are lack of schema yet are able to paint very well following another drawing, may not be able to paint by following an object." [2]

\section{KANT'S SCHEMATISM}

The word schema (Schema) orig inates from ancient Greek $6 \times \eta \mu \alpha$, original meaning was image and appearance, then transferred to portray of the most basic features, or sketch, outline, abstract graphics, etc. It is schema in Latin, meaning is essentially unchanged. In German, the word has multiple meanings, its interpretation in Duden German Dictionary is: 
Haltung (attitude), Stellung (postures), Gestalt (form, namely the so-called Gestalt), Figure (shape), Form (form), these are the usage in everyday language. It has not gained special philosophical significance before Kant, Kant introduced the word into philosophy, making it appear in the context of transcendental philosophy, thus becoming a philosophical concept with special significance.

\section{A. Raising Background of Schematism}

The history of Western philosophy has occurred two ways of thinking regarding the understanding of different nature or different classes, especially in particularity and generality, intuitive and conceptual problems: one is empirical inductive way, namely empirical concept comes from individual experience and is acquired through experience induction. Such as "a circle comes from a plate", the concept of circle is generated directly from the empirical thing-round plate, therefore, the links between the concept of circle and individual plate is based on the experience itself, no "medium" is required between them. Another way is rational interpretation way based on the links between particularity and generality, namely general concept deduces individual concept by logical relationship, which is based on inclusion of "scope" concept in "species" concept. This link between individual and general concepts is based on rationality itself, "medium" is also not required. Among these two ways, the former is deducing generality with particularity, the latter is deducing particularity with generality, both are empirical or rational, although they can link generality with particularity, it seems a link of homogeneous understanding to Kant. The former concept of experience is homogeneous or similar to experience itself, both have a common existence, that is experience. The latter borrows logic to introduce particularity from the concept of generality, both are also homogeneous, either general or individual concepts all take rationality as the foundation.

The links of heterogeneous understanding raised by Kant refers to the pure intellectual conceptual scope as the main form of subjective thinking, which is a miscellaneous link between the understanding contents of heterogeneous sensible intuition. The former is the general form of subject, the latter are the object contents, in Kant's mind, the links between the two are a linking is sue of a different nature or different classes. Kant has proved in scope transcendental deduction that the pure intellectual sensibility of scope does not come from sensibility, but from intellectuality itself. And heteropoly is related to foreign bodies ("thing-in-itself") as the source of phenomenon material. Such sensible intuition and intellectual concepts are heterogeneous class from different sources. But how to conclude the phenomenon of special phenomenon under the scope of purely intellectual concept, and how to link particularity with generality is the immediate cause that Kant raised schematism.

\section{B. Characteristics of schematism}

Pure concept (scope) is innate, which means it derives from thinking subject itself instead of from experience and exists as innate knowledge; pure concept is transcendental, which means it can not do without experience as a thinking form of subject, and it develops only with experience and opportunity, it means nothing without experience, and it is an innate condition for experience to be possible; pure concept is objective, which means it has generality and inevitability, and is a concept used as regularity and orderliness; pure concept is defined, which means that scope shall conclude sentimental miscellaneous materials under a defined transcendental form, namely concluding in self transcendental unification, and thus the concept of experience can become possible. The function of pure concept means that integrated and unified judgment conducted by subject through this form of thinking cannot only integrate and unify representation of concept judgment, but also unifies representation in intuition. But judgment does not come from pure conceptual category, but comes from transcendental ego; ego is judged through pure concept, therefore, judgment is the function of integration and unification by subject or basic mode of logical function by subject. Actually, Kant thought from transcendental logic meaning, integration and unification of conceptual category for miscellaneous materials is ego judgment function of cognitive logic subject. Then, pure concept is not only trans cendental, but also logical concept as well unification of both. This shows that, pure concept as ego thinking mode of pure concept only can play its functional value and can show subject meaning in unification of subject and object when combined with understanding contents in cognition. Apriority in Kant's category shows that subject exists prior to object of experience, as thought rule and logic structure composed of knowledge, which is the basis of knowledge being knowledge; while schema of category means that this congenital knowledge can be connected with sensibility materials and become regular scientific knowledge in certain structure.

Image picture gives people specific image, while schema means that object realizes construction purpose of subject according to requirements of subject and forms specific conceptual knowledge. Schema should present conceptual specialization, make pure abstract concept realize specific visualization, that is, a method of creating new knowledge according to rule require ments of subject form being applied to object contents and object contents following subject form. Schema of triangle is not specific triangle such as right angle, acute angle and obtuse angle, but is a method of representing how triangle concept becomes specific triangle, therefore, schema is generality mode with sensibility structure expressed by imagination according to demand of features, laws and subject in the subject's imagination. This is not composition of specific triangle, but is an image method of constructing general triangle with generality. Schema is not a concept which can be grasped, but gives graphics structure rule of triangle concept and intuitively as sociate general triangle and a determined triangle in imagination according to the subject's intention and forms a sensibility pattern with generality.

\section{Meaning of the Schematism}

The transcendental schematism of Kant has its important value and meaning in the history of western philosophy and human's cognition his tory.

First, the schematism finds the new link method for the unification of subjective and objective bodies, breaks through the traditional theory which only uses the cognition of the 
same kind to deal with the unification of subjective and objective bodies in the history of the western philosophy. In history of the western modern philosophy, for empiristic philosopher, the unification of the individual and the subject with the object is done by empirical induction means, and there will be no knowledge without the experience. It shows always in rigid constitution. However, the rationalism philosopher investigates the unification of the subject and the object from the logical analysis, and there will no knowledge without the reason. Theses two kinds of unification and link of the subject and object, have a certain reason for themselves, and the schematism of Kant embodies exactly the comprehensive tendency of such two kinds of theories, overcoming the shortage of the empiricis $m$ and rationalis $m$ in a certain degree. In dealing with the unification or lin $\mathrm{k}$ between the subject and object, Kant protrudes the time. As the time has duality of sensitivity and intellectuality, it then becomes the bridge of link between the subject and object, the individual and generality. It is very meaningful for Kant to put the unification of subject and object in the time. The succession and onedimensionality make the unification of subject and object become into the process. The schema has determined a new type for the unification process of subject and object. The subject and object are just put into this special structural type before the interaction of the two can be realized for unification.

Second, the schema is the form for realizing the jump of cognition. Schematism reveals a kind of constructive mode of the subject to the object, thus forming general and inevitable scientific knowledge. Such a construction expresses a kind of comprehensive activity, and Kant stresses the transcendental synthes is of the imag ination. He said, "Hence it is now more apparent that intellectual schema indicates that the transcendental consolidated results via imagination does not lie on other things, but only for unification of intuitive diversity in inner sense, which, indirectly the unification of apperception of corresponding functions of inner sense organs (sensitivity)". Imagination will set up the systematicness in the miscellaneous material, and such unification of the systematicness will act on the diversity of the intuitive feeling directly, making the diversity set up the unified order. The real source of such unification is the primitive comprehensive unification of the transcendental apperception or the transcendental unification of the self-awareness. Such unification of the transcendental apperception must put the unification into the phenomena by a kind of ability of the subjective self, and such ability of subjective self is imagination. The comprehensive actuality of the imagination is the internal power in the unification of subject and object. Kant thought that, without the imagination, the constructive activity of scientific knowledge will not have power to realize it.

Thirdly, the schema limits the application of scope, and make the intellectuality to become in reality. The schema takes the time as basis, and mixes to enter the time to make the time have sensibility. When scope is introduced into the time, it expresses a kind of schema of time. As the sensibility has a restriction to the time schema, the scope will be restricted too in the time by the sensibility. The schema causes the scope and phenomena to be harmony and consistent, it is exactly the sensibility restricting action to the scope make the scope keep the application in the phenomena. Kant thought the scope of number is the quantity of phenomena, the scope of "one", "many" and "whole" is restricted by the sentience of the time in the schema, so it can become into real knowledge. If excluding the restriction of the schema to the scope, the scope will have no perceptual condition, and only have the hollow and pure concept. Although it has logic meaning, it can not make the intellectuality become knowledge. For example, the perceptive conditions such as scope of number, entity and so on, are only the subject without the perceptive conditions such as quantity, durability and so on; only when the scope combines with perceptive materials in the scope, intellectuality can become real knowledge. Kant said, "therefore, the scope has no schema, and only has the function of intellectuality for concept, and will not express the object. Such a meaning makes it come from the sensibility. The sensibility makes the intellectuality be realized in the restriction process to the intellectuality". This shows that, the scope can not do without the sensitivity and its restriction in the schema, and it is the schema that restricts it and makes the constructive object of the intellectuality become knowledge.

Fourthly, the schematism has the meaning of integrated subjectivity finality and dialectics. Kant established the important position of subjective self in the epistemology, actually establishing the status of human being. The integrated subjectivity finality of the schematism mainly shows in the two aspects, one is to establish the universal and inevitable scientific knowledge, and the other is to establish the philosophical basis for the possibility of the scientific knowledge.

\section{CONCLUSION}

The schematicism of Kant is to deal with is sues of link and unification between the individual and the general, subject and object of the epistemology by using a new kind of theoretical form. Such theory breaks through the combination type of the traditional theory which is simplified and rigidified in dealing with such issues, making the development of epistemology go along the road of initiative and dialectics. Therefore, the schematicis m of Kant has an important value and meaning for the development of the epistemology, and also find a new theoretical method for realizing the value of the subjective self. Meanwhile, it also penetrates in the modern science, providing a certain philosophical enlightenment for the scientific development.

The current meaning of the schematicis $\mathrm{m}$ is mainly lying in aspect of the epistemology. If we can explore the issue of schema deeply on basis of the achievements of the former people, and introduce it into the schematicis $m$ of the art education, it is doubtless to have a lot of benefits. The brain of human beings is not blank at all, and the art education workers shall be sure about this point. The content of the painting comes from the cognition of the author to the objective world. Just because of the existence of the schema, the author can express the cognition to the objective world in his or her works in a certain limit 


\section{REFERENCES}

[1] "Art and Illusion" by E.H. Gombrich [UK], translated by Yang Chengkai, Li Zhengben and Fan Jingzhong, [M] Guangxi Arts Publishing House, Nanning, 2012, Page 129

[2] "Art and Illusion" by E.H. Gombrich [UK], translated by Yang Chengkai, Li Zhengben and Fan Jingzhong, [M] Guangxi Arts Publishing House, Nanning, 2012, Page 127

[3] “Critique of pure reason", Hamburg, in 1956, Page 203, A145, B185; referring to the translation version by Lan Gongwu, Page 146. 